\title{
CASE OF CUTANEOUS LEISHMANIASIS OF THE LID*
}

\author{
BY \\ GWYN MORGAN \\ Department of Pathology, Institute of Ophthalmology, University of London
}

THE lid is involved only in about 2 to 5 per cent. of cases of cutaneous leishmaniasis (Pestre, 1955), probably because the movements of the lids prevent the fly-vector of the disease from biting the skin in this region.

The typical lesion is a solitary ulcerating granuloma which starts as a small itching papule and then becomes scaled, crusted, and finally ulcerated. The causative organism is the protozoon Leishmania tropica transmitted to humans by the sandfly (Phlebotomus), the disease occurring naturally in dogs and cats, and in rodents in Turkestan. Timpano (1946) has suggested that the gnat may also be able to transmit the condition.

Scrapings from lesions, especially early ones, show Leishman-Donovan bodies which are found mainly within histiocytes, but also extracellularly. The parasitized histiocytes often measure $20 \mu$ or more in diameter and the bodies, which are round or oval in shape, vary between 2 and $4 \mu$ in diameter. They are non-encapsulated and consist of a relatively large peripherally placed nucleus and a small rod-like or oval paranucleus set at a tangent to the nucleus. This paranucleus, also called kinetoplast or blepharoplast, produces the flagella when the protozoon changes into the flagellate form outside the body. Leishman-Donovan bodies are best stained by Giemsa's stain, both the nucleus and paranucleus appearing bright red. The presence of these bodies from a typical skin lesion was first described by Cunningham in 1885 , who noted deeply staining parasitic bodies in mononuclear cells. The first accurate description of the causative parasite was made by Borovsky in 1898, and in 1902 Shulgin suggested that some night-biting insect was the intermediary host. In 1908, Marzinowsky inoculated himself in Moscow with material obtained from the Caucasus, and reproduced a typical sore on his forearm.

Cutaneous leishmaniasis of the lid may also occur in mucocutaneous leishmaniasis due to $L$. braziliensis, the infected material reaching the lid from nasal mucosal lesions via the nasolacrimal duct (Machado, Machado, and Moura, 1958).

Although cases of cutaneous leishmaniasis of the lid have appeared in the foreign literature (e.g., Frezzotti, 1955; Pestre, 1955; Rende, 1956), the present case is the first to be described in the English literature.

* Received for publication September 30, 1964. 


\section{Case Report}

An 18-year-old English girl complained of a lesion of the right upper lid and outer margin of the eyebrow which had been present for one month. It had appeared as a small, itching, papule two months after she had returned to England from a holiday in Majorca. On examination the lesion was raised, firm, dome-shaped and dull red in colour (Fig. 1).

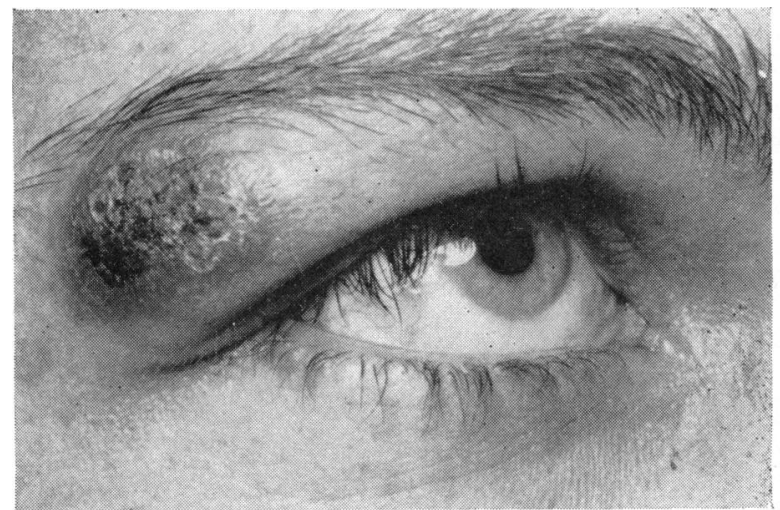

FIG. 1.-The right brow and eyelid, showing a raised, crusting, scaling nodular swelling.

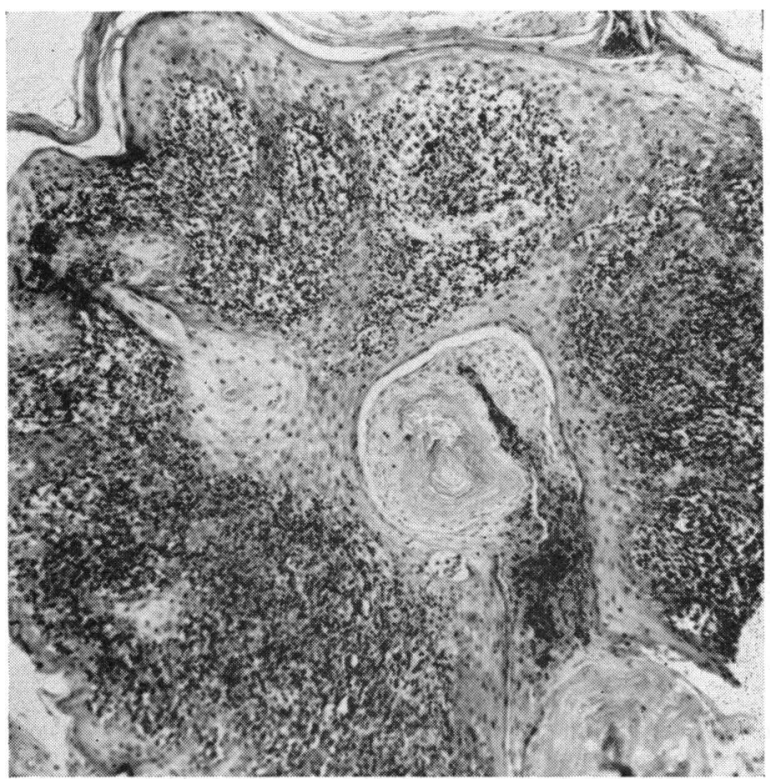

Fig. 2.-Low-power view of the lesion showing irregular acanthosis and a marked inflammatory infiltrate in the epidermis and dermis. Haematoxylin and eosin, $\times 55$.

Sections of a biopsy showed irregular, keratotic, acanthotic squamous epithelium overlying a granulomatous reaction in the dermis consisting of lymphocytes, plasma cells, occasional eosinophils, and histiocytes. Many of the latter contained Leishman-Donovan bodies, which were also seen extracellularly (Figs 2, 3, and 4). 


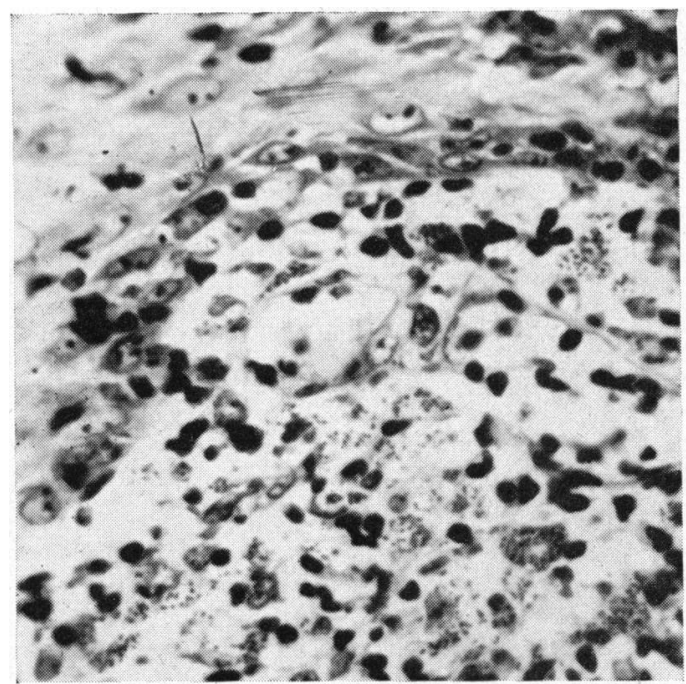

FIG. 3.-High-power view showing a polymorphous collection of inflammatory cells and parasites in the epidermis and dermis. Haematoxylin and eosin, $\times 415$.

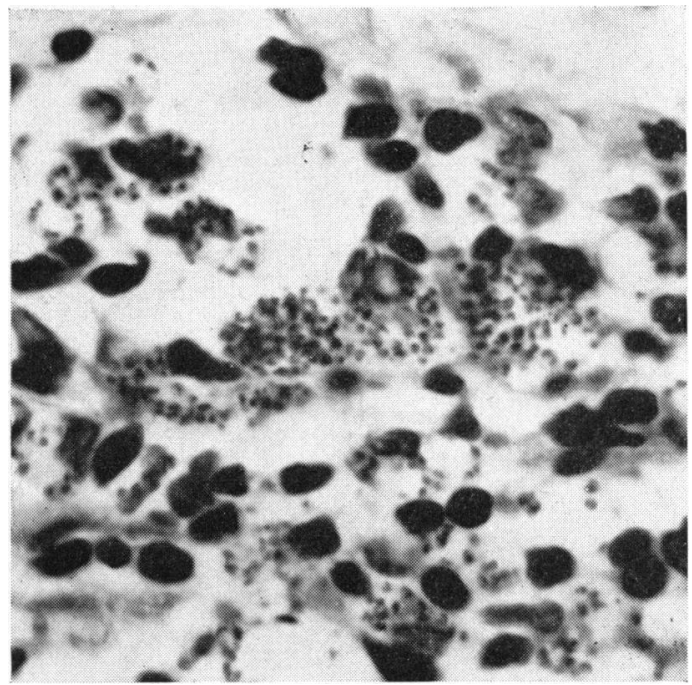

FIG. 4.-High-power view showing intracellular and extracellular Leishman-Donovan bodies in the dermis. The characteristic nucleus and paranucleus are clearly seen in the majority of the parasites. Giemsa, $\times 910$.

Before making the diagnosis of leishmaniasis, it was necessary to exclude two diseases which sometimes involve the lid, i.e., histoplasmosis and rhinoscleroma. The former disease is caused by a fungus, Histoplasma capsulatum, and is usually generalized in its manifestations. The skin, however, is sometimes affected exclusively and the lesions show a granulomatous reaction with areas of necrosis in which there are large, pale histiocytes containing rounded, encapsulated organisms. Rhinoscleroma involves the nose, lips, and upper respiratory tract. The granulomatous skin lesions contain large, rounded histiocytes, the Mikulicz cells, which measure $100-200 \mu$ in diameter and contain Gram-negative rods known as Frisch bacilli. Another prominent feature is the presence of Russell bodies, which are never seen in cutaneous leishmaniasis. The skin lesion in this case, therefore, was neither histoplasmosis nor rhinoscleroma, but quite clearly leishmaniasis.

Treatment.-Daily intramuscular injections of sodium stibogluconate in divided doses $(20 \mathrm{mg}$. per kg. body-weight) were given for two weeks. This drug is a quinquivalent antimony derivative containing 30-34 per cent. by weight of antimony. There was an excellent response and at the end of this period the lesion had disappeared, except for a small area which required local injections of the same drug daily for a further five weeks, when it healed completely.

\section{Discussion}

The incubation period of cutaneous leishmaniasis varies between several weeks and about a year. As already stated, the causative organism is the parasite $L$. tropica, though, on occasions $L$. braziliensis may be involved. These two organisms, together with $L$. donovani, the causative organism of kala-azar, are morphologically indistinguishable, but may be differentiated by immunological methods.

Cutaneous leishmaniasis is endemic within certain limited areas in many warm climates. In Italy, Vanni (1938) described 300 cases involving various exposed parts of the body. The condition is common in Morocco, the Sahara, Egypt, Crete, Cyprus, Sicily, Syria, Palestine, the Sudan, Nigeria, Iraq, Persia, the Caucasus, India, Turkestan, China, and South and Central America. 
Although it may occur in areas where kala-azar is endemic, its distribution as a rule is quite distinct. The two diseases, of course, may occur in the same patient, and recovery from one does not necessarily protect from subsequent attacks of the other. In the tropics, the cutaneous disease is prevalent about the beginning of the cool season; in more temperate climates, however, the prevalence is towards the end of the summer or early autumn.

Wenyon (1911) was the first worker to suggest that the sandfly (Phlebotomus) was the vector of the disease in Baghdad. Later, confirmatory evidence was supplied by Sergent, Sergent, Parrot, Donatien, and Béguet (1921), who produced the lesion in Algiers after scarifying the skin of volunteers and applying a saline suspension of crushed Phlebotomus papatasii flies which were obtained from Biskra, more than 350 miles from Algiers where the disease did not occur. In this series, the incubation period was two and a half months. In 1941, Adler and Ber produced 28 lesions in 5 volunteers by the bites of infected sandflies.

When the skin of the lids is affected, both upper and lower lids may be involved on either their outer or inner aspects. When the outer aspect is involved, the disease is usually self-limiting, healing taking place by scarring in up to one year. Pestre (1955) has described lid lesions resembling chalazion. A much more serious train of events is to be expected when the inner aspect of the lid is involved. Secondary infection with destruction of underlying soft and bony tissue is common. Stenosis of the lacrimal duct leads to a chronic discharging fistula and a secondary conjunctivitis and uveitis may be seen. Scuderi (1947) has pointed out that the conjunctivitis is due to secondary invading organisms. If the patient is seen at this stage it may be extremely difficult to distinguish the condition from lupus vulgaris or syphilis. In a small percentage of the older lesions, at the site of a primary sore which has practically healed (Kochs, 1955), brownish-red nodules resembling those seen in lupus vulgaris may form. Histological examination of the lesion reveals a tuberculoid infiltrate which is indistinguishable from that of lupus vulgaris. Caseation is absent and Leishman-Donovan bodies are difficult to detect.

\section{Summary}

A case of cutaneous leishmaniasis of the lid is described. The lesion, which was contracted in Majorca, was treated successfully with an antimony compound, sodium stibogluconate. The aetiology and pathogenesis of the disease are discussed.

I should like to thank Dr. Peter Borrie for his permission to publish this case and Professor Norman Ashton for his valuable advice. I am grateful to Miss E. M. FitzGerald for secretarial help, and to Mr. F. H. Vincer and Mr. V. J. Elwood for their technical assistance in the preparation of sections and photomicrographs.

\section{REFERENCES}

AdLer, S., and Ber, M. (1941). Nature (Lond.), 148, 227.

Borovsky, P. (1898). Quoted in “Manson's Tropical Diseases”, ed. P. H. Manson-Bahr, 13th ed., 1950, p. 177. Cassell, London.

CunNinghaM, D. D. (1885). Ibid., p. 177.

Frezzotti, R. (1955). G. ital. Oftal., 8, 312.

Kochs, A. G. (1955). Arch. Derm. Syph. (Berl.), 199, 540.

Machado, N. R., Machado, J. G. De Castro, and Moura, P. Alcover de (1958). Rev. bras. Oftal., 17, 279. 
Marzinowsky, E. J. (1908). Z. Hyg. Infekt.-Kr., 58, 327.

Pestre, A. (1955). Algérie méd., 59, 589.

RENDE, S. (1956). Riv. ital. Tracoma, 8, 148.

SCUDERI, G. (1947). Rass. ital. Ottal., 16, 335.

Sergent, Ed., Sergent, Et., Parrot, L., Donatien, A., and Béguet, M. (1921). Quoted in "Manson's Tropical Diseases", ed. P. H. Manson-Bahr, 13th ed., 1950, p. 179. Cassell, London.

Shulgin, K. (1902). Ibid., p. 177.

Timpano, P. (1946). Quoted by Rende (1956).

VANNI, V. (1938). Ann. Igiene., 48, 520.

WENYON, C. M. (1911). J. trop. Med. Hyg., 14, 103. 